\section{Low-noise synthesis of microwave signals from an optical source}

J.J. McFerran, E.N. Ivanov, A. Bartels, G. Wilpers, C.W. Oates, S.A. Diddams and L. Hollberg

\begin{abstract}
The low-noise synthesis of a harmonic comb of microwave frequencies using a $1 \mathrm{GHz}$ femtosecond-laser-based synthesiser that is referenced to a cavity-stabilised laser is demonstrated. The residual phase noise is $\sim 110 \mathrm{dBc} / \mathrm{Hz}$ at $1 \mathrm{~Hz}$ offset from the $10 \mathrm{GHz}$ harmonic. Phase noise is measured with an interferometric measurement system having low sensitivity to $\mathrm{AM}$.
\end{abstract}

Introduction: Low phase noise microwave sources are important in a variety of scientific and technological fields, including radar and remote sensing, communications and navigation, high speed electro nics, and the development of atomic frequency standards. At present, the lowest noise microwaves sources are synthesised from quartz oscillators or generated with dielectric or metallic resonant cavity oscillators $[1,2]$. However, a cavity in the optical domain can have a resonance $Q \quad v / \Delta v \geq 10^{11}$, which leads to exceptional short term frequency stability. For example, a laser at $532 \mathrm{THz}$ stabilised to an isolated Fabry Perot optical cavity has demonstrated fractional frequency stability near $4 \times 10^{16}$ ( 1 10 s averaging) and a linewidth below $0.2 \mathrm{~Hz}$ [3]. This corresponds to single sideband phase noise spectral density (PSD) of $\mathcal{L}_{\phi}(f) \sim 17 \mathrm{dBc} / \mathrm{Hz}$ at $f 1 \mathrm{~Hz}$ offset frequency from the optical carrier. Assuming ideal division of the optical carrier by $N 53,200$ this would result in a corresponding $10 \mathrm{GHz}$ signal with $\mathcal{L}_{\phi}(1 \mathrm{~Hz}) \quad 112 \mathrm{dBc} / \mathrm{Hz}$, which is $\sim 50 \mathrm{~dB}$ better than that achieved with the best conventional microwave sources.

Femtosecond laser optical frequency combs (FLFC) have emerged as a new optical to microwave frequency divider [4]. The optical output of a mode locked femtosecond laser consists of an array of phase coherent optical frequencies that are separated by the pulse repetition rate $f_{r}$ (see Fig. 1). The frequency of the $n$th mode of the comb is given by $f_{n} \quad n f_{r}+f_{o}$ where $f_{o}$ is the carrier envelop offset frequency common to all modes. When phase locked to an optical source, $f_{\text {opt }}$, the FLFC provides a repetitive train of optical pulses at a subharmonic of the optical frequency. However, attempts to extract low noise electronic signals via photodetection of the optical pulses were hampered by the excess phase noise associated with the photodetection process [5]. Having further reduced several sources of noise we demonstrate here the synthesis of microwave signals at $10 f_{r} \quad 10 \mathrm{GHz}$ with residual noise near $110 \mathrm{dBc} / \mathrm{Hz}$ at $1 \mathrm{~Hz}$ from the carrier and $160 \mathrm{dBc} / \mathrm{Hz}$ at an offset of $1 \mathrm{MHz}$. Because this low level of noise was present on a carrier having relatively low power of $15 \mathrm{dBm}$, an interferometric, carrier suppressed noise measurement system (NMS) with low residual ampli tude sensitivity was employed [6].

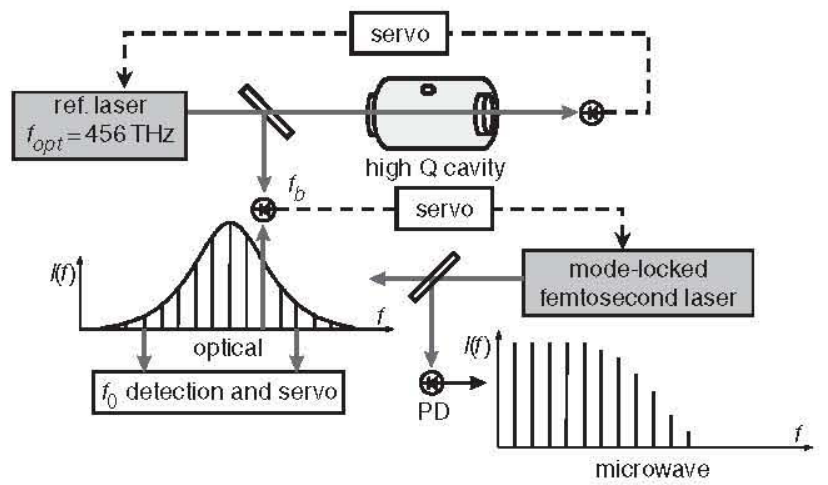

Fig. 1 Schematic of femtosecond laser frequency comb (FLFC)

Microwave synthesis and measurement: Microwave signals are generated from two separate $1 \mathrm{GHz}$ FLFCs producing optical spectra extending from 580 to $1080 \mathrm{~nm}$ (at $50 \mathrm{~dB}$ below maximum) $[7,8]$. Phase stabilisation of the FLFC requires two control loops, as shown in Fig 1. The offset frequency, $f_{o}$, of the FLFC is measured using a $2 \mathrm{f} 3 \mathrm{f}$ self referencing technique [8], and is then phase locked to a stable RF reference. Additionally, the nearest mode $(n \simeq 456,000)$ of the FLFC is phase locked to a cavity stabilised diode laser at $f_{\text {opt }} 456 \mathrm{THz}(657 \mathrm{~nm})$ with a small RF offset $f_{b}$. A second inde pendent FLFC is controlled in the same manner. The net result is that the repetition rates i.e comb spacings) of each of the two FLFCs are equal and phase coherently connected to the stable $456 \mathrm{THz}$ optical reference as $f_{r} \quad\left(f_{\text {opt }}+f_{o}+f_{b}\right) / n$.

The pulse trains of the lasers with $f_{r} \quad 1 \mathrm{GHz}$, are incident upon high speed $(>10 \mathrm{GHz})$ photodetectors $(\mathrm{PD})$ and the 10th harmonics of the repetition frequencies are selected with bandpass filters for phase noise measurements. InGaAs and GaAs PDs were used for respective FLFCs, and the power in the $10 \mathrm{GHz}$ harmonics was approximately $15 \mathrm{dBm}$ (2.6 $\mathrm{mA}$ average photocurrent). Phase noise was measured with the interferometric NMS shown in Fig. 2. It features a microwave inter ferometer followed by a low noise amplifier and a nonlinear mixing stage based on a double balanced mixer (DBM). The latter serves as a phase detector for measuring the difference in the phase fluctuations of $10 f_{r}$ from the femtosecond lasers. Phase synchronisation of the two femto second pulse trains results in the destructive interference of the extracted microwave signals at the dark port of the microwave interferometer (output of a three way power combiner in Fig. 2). The residual fluctua tions of the difference signal from the dark port of the interferometer are amplified and down converted to DC where their spectral density is measured with a FFT spectrum analyser. To improve the resolution of the spectral measurements the carrier of the difference signal is suppressed (by adjusting the insertion loss in one of the interferometer arms). This ensures a small signal operation of the microwave amplifier. Having a high gain low noise amplifier in front of the mixing stage reduces the effective noise temperature of the entire measurement system from more than $10^{4} \mathrm{~K}$ to almost ambient temperature [6], thereby, permitting noise measurements with sensitivity approaching the standard thermal noise limit.

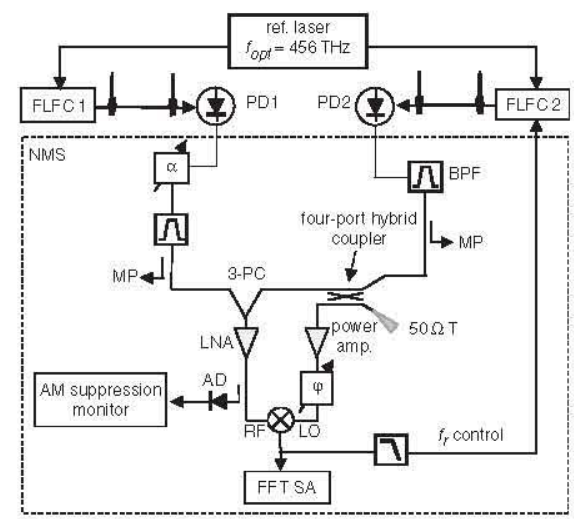

Fig. 2 Phase noise measurement system (NMS) for $10 \mathrm{GHz}$ signals obtained from two FLFCs

An imperfect tuning of the NMS makes it sensitive to amplitude fluctuations of the input signal. To avoid misinterpretation, the ampli tude sensitivity of the NMS must be minimised by varying the phase difference between the signals entering the input ports of the DBM. After course adjustment of the phase difference to locate minimum AM sensitivity, part of the DBM output acts a phase discriminator in a very low bandwidth $(\sim 3 \mathrm{~Hz})$ servo system adjusting the phase of the RF frequency offset $f_{b}$ used in the control of FLFC 2 (see Fig. 2). The action of this low bandwidth servo system is accounted for post PSD measurement. Out of loop phase fluctuations in the FLFCs at the level of $\sim 100 \mu \mathrm{rad} / \mathrm{s}$ at $10 \mathrm{GHz}\left(\sim 1 f_{s} / s\right)$ are corrected for with the low bandwidth servo. These fluctuations are believed to arise from temporal variations of path lengths and $\mathrm{AM}$ to $\mathrm{PM}$ conversion in the photodiodes.

Results: An example of the measured phase noise assuming equal contributions from each FLFC is given in Fig. 3 (curve (i)). The results demonstrate that when referenced to an optical signal (e.g. from a $\mathrm{Hg}^{+}$or $\mathrm{Ca}$ optical cavity) the FLFC used here can produce microwave signals with phase noise $40 \mathrm{~dB}$ lower than commercial microwave oscillators for Fourier frequencies between 1 and $300 \mathrm{~Hz}$. Integration of the phase noise over the full measurement range yields a relative timing jitter of only $0.8 \mathrm{fs}$. 


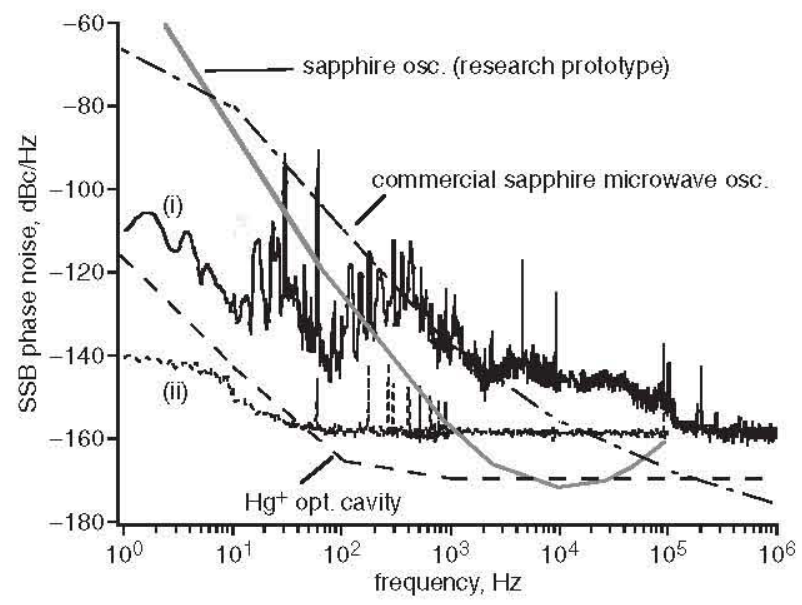

Fig. 3 Residual phase noise on $10 \mathrm{GHz}$ harmonic of phase stabilised femtosecond laser frequency comb (curve (i)) compared to other low phase noise microwave sources

Curve (ii) is noise floor of measurement system

Better attention to ground loops and shielding of the microwave interferometer components may remove several of the bright lines corresponding to $60 \mathrm{~Hz}$ harmonics seen in the measured trace. The majority of the noise between $10 \mathrm{~Hz}$ and $1 \mathrm{kHz}$ is typical of vibrations that could be transmitted to the FLFCs and optical detection systems.

Beyond $100 \mathrm{kHz}$ the measured PSD approaches the noise floor set by thermal noise of the electronics and intrinsic fluctuations of the low noise amplifier in the NMS. By increasing the available signal from the PDs, the noise floor at these high Fourier frequencies can be reduced. However, calculations suggest that we are also close to a phase noise limit set by shot noise of the detected photocurrent. For example, if we assume the PSD of the shot noise limited phase fluctuations in a single quadrature is given by

$$
\mathcal{L}_{\phi}^{\text {shot }} \simeq \frac{e i R}{2 P_{\text {signal }}}
$$

where $P_{\text {signal }}$ is the power of extracted microwave signal, $i$ is the mean photocurrent and $R$ is load impedance, we deduce that the expected noise floor arising from shot noise is $\sim 155 \mathrm{dBc} / \mathrm{Hz}$ for the signal parameters mentioned above. This shot noise analysis may be overly simplistic, since time varying light signals can produce deviations from the well known shot noise formula [9]. Improvements in photodetector power handling and sensitivity at $800 \mathrm{~nm}$ may help clarify the origin of this high frequency noise floor.

Conclusions: A carrier suppressing, interferometric phase noise measurement system has been used to measure the phase noise of frequency divided optical signals. The excess noise in the frequency division with a FLFC is $\sim 110 \mathrm{dBc} / \mathrm{Hz}$ at $1 \mathrm{~Hz}$ from the $10 \mathrm{GHz}$ carrier, enabling synthesis of microwave signals with $\sim 40 \mathrm{~dB}$ lower phase noise close to the carrier than the best reported microwave oscillators.

Acknowledgments: This research was supported by DARPA MTO. J.J.M. and E.N.I. thank the Australian Research Council and NIST for supporting them as guest researchers at NIST. This work is the contribution of an agency of the US government; not subject to copyright in the US.

J.J. McFerran, E.N. Ivanov, A. Bartels, G. Wilpers, C.W. Oates, S.A. Diddams and L. Hollberg (Time and Frequency Division, National Institute of Standards and Technology, 325 Broadway, MS 84710 , Boulder, CO 803053328 , USA)

\section{References}

1 Chang, S., Mann, A.G., and Luiten, A.N.: 'Improved cryogenic sapphire oscillator with exceptionally high frequency stability', Electron. Lett., 2000, 36, (5), pp. 480481

2 Sen-Gupta, A., et al.: 'High-spectral-purity microwave oscillator: design using conventional air dielectric cavity', Proc IEEE FCS and EFTF, 2003, pp. 423429

3 Young, B.C., et al.: 'Visible lasers with subhertz linewidths', Phys. Rev Lett., 1999, 82, (19), pp. 37993802

4 Diddams, S.A., Ye, J., and Hollberg, L.: 'Femtosecond lasers for optical clocks and low noise frequency synthesis' in Ye, J., Cundiff, S.T. (Eds): 'Femtosecond optical frequency comb technology' (Springer, New York, USA, 2005)

5 Ivanov, E.N., Diddams, S.A., and Hollberg, L.: 'Analysis of noise mechanisms limiting the frequency stability of microwave signals generated with a femtosecond laser', IEEE J. Sel. Top. Quantum Electron., 2003, 9, (4), pp. 10591065

6 Ivanov, E.N., Tobar, M.E., and Woode, R.A.: 'Microwave interferometry: application to precision measurements and noise reduction techniques', IEEE Trans. Ultrason. Ferroelectr. Freq. Control, 1998, 45, (6), pp. 15261536

7 Bartels, A., and Kurz, H.: 'Generation of a broadband continuum by a Ti-sapphire femtosecond oscillator with a $1 \mathrm{GHz}$ repetition rate', Opt. Lett., 2002, 27, (20), pp. 18391841

8 Ramond, T.M., et al.: 'Phase coherent link from optical to microwave frequencies by means the broadband continuum from a $1 \mathrm{GHz} \mathrm{Ti}$ sapphire femtosecond oscillator', Opt. Lett., 2002, 27, (20), pp. 1842 1844

9 Winzer, P.J.: 'Shot-noise formula for time-varying photon rates: a general derivation', J. Opt. Soc. Am. B, 1997, 14, (10), p. 2424 